\title{
Mental health literacy and mental health status in adolescents: a population-based survey
}

Lawrence $T \operatorname{Lam}^{1,2}$

\begin{abstract}
Background: This study aims to investigate the association between mental health literacy and the mental health status, particularly depression, among adolescents.

Methods: This was a population-based health survey utilising a two-stage sampling technique. Mental health literacy was measured by the Australian National Mental Health Literacy and Stigma Youth Survey with the depression vignette only. Depression was assessed by the Depression sub-scale of the Depression, Anxiety, Stress Scale. Data were analysed using multiple logistic regression modelling techniques with adjustment for cluster sampling effect.

Results: A total of 1678 students responded to the survey providing usable information. Only 275 (16.4\%) respondents were classified as having an adequate mental health literacy level with correct identification of depression and also intended to seek help, with 392 (23.4\%) of the total sample correctly identified the vignette as depression. Two hundred and forty eight (14.8\%) were classified to have moderate to severe depression. Multiple logistic regression analysis results suggested that young people who had experienced moderate to severe level of depression in the week prior to the survey were more likely to have an inadequate level of $\mathrm{MHL}(\mathrm{OR}=1.52,95 \%$ C.I. $=1.01-2.31)$ after adjusting for a potential confounding factors and cluster sampling effects.

Conclusions: Results suggested that mental health literacy level was associated with mental health status, particularly depression of young people. The results have important implications, both clinically and on a population level, on the prevention of mental health problems and for the improvement of the mental health status of adolescents.
\end{abstract}

Keywords: Mental health literacy, Mental Health outcome, Depression, Adolescents, Health Survey

\section{Background}

The US Institute of Medicine (IoM) 2004 report first defined health literacy as: "the degree to which individuals have the capacity to obtain, process and understand basic health information and services needed to make appropriate health decisions" [1] This definition was subsequently enriched by the World Health Organisation (WHO) in 2007 to "the cognitive and social skills which determine the motivation and ability of individuals to gain access to, understand and use information in ways which promote and maintain good health [2]."

Applying the concept of health literacy to the mental health arena, Jorm et al. have extended it and coined the term "Mental Health Literacy" (MHL) with the definition

\section{Correspondence: Itlam@ied.edu.hk}

'Discipline of Paediatrics and Child Health, Sydney Medical School, The University of Sydney, Australia, Sydney, Australia

${ }^{2}$ Department of Health and Physical Education, The Hong Kong Institute of Education, 10 Lo Ping Road, Tai Po, N.T, Hong Kong SAR, China "knowledge and beliefs about mental disorders which aid their recognition, management or prevention" [3]. He has also included the following as the characteristics of the MHL:

- The ability to recognise specific disorders;

- Knowledge of how to seek mental health-related information;

- Knowledge about risk factors and causes of mental health disorders;

- Knowledge about how to self-treat and of the availability of professional help;

- The attitudes that promote the recognition of mental health problems;

- The attitudes that promote seeking appropriate help [3].

In terms of the measurement and assessment of MHL, there has been some on-going development since the 
inception of the concept of MHL and the initial design and utilisation of the vignette-based instrument in 1995. Jorm et al. reported the use of the vignette-based method to examine the ability of individuals to recognise mental disorders or problems in the national population survey in 1995 [3]. He also reported the use of some rating scales to assess the perceptions of the respondents on a list of pharmacological and non-pharmacological treatments related to the vignettes [3]. Since then, this approach of MHL assessment has been further developed to include questions on other important aspects of the MHL concept. These include: intention to seek help; belief and intention about first aid; belief about intervention and prevention [4]. This approach of assessment on MHL has been adopted widely [5-11].

Since the inception of the MHL concept in 1995, ample studies have been conducted particularly on the assessment of MHL [12-20]. As expected, many of these studies were carried out in Australia by Jorm and his colleagues or other Australian researchers [15-20], and many others were conducted in other countries in different adult populations. For example, studies on mental health literacy among Chinese adults and elderly people had been conducted in China, Hong Kong, Australia, and Canada particularly in the area of depression and schizophrenia [18-21]. On the whole, the mental health literacy level among Chinese adults was not high [18-21]. For adolescents, studies on the MHL are far less in comparison to the adult population [22-32]. Among the studies on the MHL of adolescents in the last decade, most were conducted in older adolescents [26-29], such as university students, student nurses, and rural young people [30-33]. Some studies involved both younger and older adolescents [22-25], but very few were found on the MHL of younger adolescents in junior and senior high schools [23]. The majority of these studies mainly concentrated on the assessment of the mental health literacy level and the associated characteristics such as attitudes towards help seeking and stigmatisation [22-27,32,33].

Among the studies on MHL in adolescents, some also examined factors that were associated with MHL. Leighton's study in 2010 reported that socioeconomic disadvantage and low levels of educational attainment were not associated with inadequate MHL, however, it was found that females and those with experience of mental health problems were more likely to seek help from different sources [24]. Being a female, of older age, and having a higher level of education were also found to be related to the ability to recognise depression in a vignette [29]. In a study among university students with different ethnic backgrounds, it was revealed that Chinese female students had a comparatively better knowledge of the symptoms of depression when comparing with their Malay and Indian counterparts [27].
In terms of the relationship between MHL and mental health outcomes, more specifically whether an inadequate level of MHL would be associated with an increased risk of mental health problems, there is yet a study to be found in the literature. Hence, this study aims to investigate the relationship between mental health literacy and the mental health status, particularly depression, in a population of younger adolescents in junior and senior high schools.

\section{Methods}

This study was a population-based cross-sectional health survey utilising a two-stage random cluster sampling design. The study was conducted in Nanning city of the Guangxi Province in the South Western region of China in October 2013. Nanning, the capital city of the Guangxi Province, is the biggest and most populated city of the Province with an estimated population of about 6.7 million in 2010. The population size for young adolescents aged between 15 and 19 years was estimated to be 505677 . This represented about $7.6 \%$ of the total population in the city. Institute ethics approval for the study was granted by the Human Ethics Committee of the Hong Kong Institute of Education.

The sample consisted of high school students aged between 13-17 years with the total student population attending high schools in the designated region as the sample frame. The local education department provided a list of high schools located within the boundary of the school district for sampling. The sample was generated using a two-stage random cluster sampling technique. First, using individual schools as the primary sampling unit, a number of schools were randomly selected with a probability proportional to the size of the target population. Second, using the class as the secondary sampling unit, different clusters of students were randomly selected from each grade of the selected schools. Participants were recruited from 12 high schools and 48 different classes.

The health survey was conducted within two weeks on campus at different schools. Students and parents from the selected classes from different schools were informed of the survey via a written information letter. They were invited to participate in the study and wilful consent was implied by the filling in of the questionnaire. During the survey students were asked to fill in a self-reported questionnaire designed specifically for the study.

Mental health Literacy was assessed using questions adopted from the Australian National Mental Health Literacy and Stigma Youth Survey [25]. The Youth Survey questionnaire was designed by Jorm et al. in accordance to his MHL framework. In this study, the depression vignettes with the associated questions were used. These questions covered the following areas: recognition of disorder/mental health problem; intended actions to seek help and perceived barriers; beliefs and intention about first aid; 
beliefs about interventions; beliefs about prevention; stigmatising attitudes and social distance; exposure to mental disorders. At aforementioned, the MHL instrument was validated and widely used in many studies and different countries. Permission to use the Youth questionnaire in this study has been granted by Jorm and his team. Since MHL is a rather complex construct consisting of multiple dimensions, the level of MHL should not be simply assessed by a single dimension such as the recognition of mental health problem. In order to enhance the validity of the exposure measure, a composite variable was created combining two important dimensions of the MHL construct, namely recognition of mental health problem and the intended actions to seek help. The justification for choosing the two dimensions, namely the recognition of disorders and the intention to seek help, of the MHL construct instead of the others was based the definition of the MHL defined by Jorm et al. [3] As stated, the main features of MHL is knowledge and beliefs about mental disorders which aid their recognition, management or prevention [3]. Hence, in terms of the adequacy of the $\mathrm{MH}$, recognition of disorder should be considered as fundamental. However, merely recognising a disorder was insufficient to fully reflect the concept of MHL without the component on the intentionality of managing the problem. Hence, a correct identification of the mental health problem with an intention to seek help was defined as having an adequate MHL level and all else were defined as inadequate.

Mental health outcomes of the study, namely depression, was assessed using the Depression sub-scale of the Depression, Anxiety, Stress Scale (DASS) [34]. The DASS is a fully validated and commonly used instrument designed for the assessment of stress, depressive symptoms, and anxiety with good psychometric properties including strong reliability and validity [34]. It has also been recommended to be used among children and adolescents [35]. As suggested by the authors of the scale, the DASS was designed as a quantitative measure of distress along three axes, however, it was not meant to be categorical assessment of clinical diagnosis [33]. Nevertheless, the scale could be useful for identifying individuals who were of high risk of mental health problems.

Information collected in the survey included demographics, whether the respondent was a single child, parental education levels, parental occupations, whether the respondent was living with parents, as well as the respondent's physical health status in the last 3 months. Data were also collected on some health behaviours, such as duration of sleep and physical activities, since they had been well-established to have an impact on depression among adolescents. Physical activities were measured using the self-reported number of days per week of moderate to vigorous physical exercise and the duration of the exercise. In accordance to the WHO recommendations for adolescents, adequate physical activity was defined as involvement of moderate to vigorous exercise for at least 60 minutes daily [36]. For sleep duration, it was calculated from the actual reported time-to-bed and time-to-wake taking away any time awoke from sleep during the night.

Data were analysed using the Stata V10.0 statistical software program. Since the study was of a cluster sampling design, data were set up with the survey design function utilising the svy commands for handling the cluster sampling effect. As aforementioned, the individual schools and classes were used as the primary and secondary sampling unit in the setup of the dataset. For the sampling weight, the total number of students in the population representing the study participants was used as the selection probability. Bivariate analyses were conducted to examine the unadjusted relationships between variables of interest, MHL, and depression. The majority of variables of interest were categorical or ordinal by nature. In terms of the exposure variable, MHL was categorised into a binary variable of two categories, the adequate and inadequate MHL levels, for ease of analysis. Depression was also categorised according to the cut-off provided by the authors [34]. Again, for ease of analysis, the variable was dichotomised into normal/mild and moderate/severe groups. Further multivariate analyses were conducted using multiple logistic regression modelling technique with adjustment for the cluster sampling effect. All significant variables identified in the bivariate analysis for depression were included in the initial regression model to be considered as potential confounders. Non-significant variables in the model were removed through a backward step-wise procedure except the exposure variable. Interaction terms between the exposure variable and other significant variables retained in the model were also tested for the examination of any effect modification. A significance level of $1 \%$ was used for testing the interaction effect and 5\% for other hypotheses.

\section{Results}

A total of 1678 students responded to the survey providing usable information. This represented a response rate of $98 \%$. Information on the characteristics of the respondents and the outcome variable, namely depression status, were summarised in Table 1. As shown, nearly $15 \%$ ( $n=248,14.8 \%)$ of the respondents could be classified as exhibiting moderate to severe symptoms of depression.

Descriptive information on major MHL variables was also summarised in Table 2 . Slightly less than a quarter $(\mathrm{n}=392,23.4 \%)$ of the sample could be able to recognise symptoms depicted in the vignette as depression. However, as they were asked whether they would seek help if they had a problem as depicted, nearly $68 \%(\mathrm{n}=1128)$ of the sample indicated an intention to seek help. For mental 
Table 1 Frequency (\%) of the characteristics of study participants $(\mathrm{N}=1678)$

\begin{tabular}{|c|c|}
\hline Characteristics & Frequency $(\%)^{*}$ \\
\hline \multicolumn{2}{|l|}{ Demographics } \\
\hline \multicolumn{2}{|l|}{ Sex } \\
\hline Male & $826(49.8)$ \\
\hline Female & $832(50.2)$ \\
\hline \multicolumn{2}{|l|}{ Age group } \\
\hline$<14$ years & $879(52.4)$ \\
\hline 14 years or older & $799(47.6)$ \\
\hline \multicolumn{2}{|l|}{ Single child } \\
\hline Yes & $714(42.7)$ \\
\hline No & $960(57.3)$ \\
\hline \multicolumn{2}{|l|}{ Family structure } \\
\hline Living with biological parents & $1436(85.7)$ \\
\hline Others & $239(14.3)$ \\
\hline \multicolumn{2}{|l|}{ Father's education level } \\
\hline University or above & $261(15.7)$ \\
\hline High school/post-secondary & $1190(71.6)$ \\
\hline Others & $212(12.7)$ \\
\hline \multicolumn{2}{|l|}{ Mother's education level } \\
\hline University or above & $180(10.8)$ \\
\hline High school/post-secondary & $1181(71.1)$ \\
\hline Others & $301(18.1)$ \\
\hline \multicolumn{2}{|l|}{ Father's Occupation } \\
\hline Professional & $354(21.7)$ \\
\hline Non-professional & $392(24.0)$ \\
\hline Others & $886(54.3)$ \\
\hline \multicolumn{2}{|l|}{ Mother's Occupation } \\
\hline Professional & $239(14.6)$ \\
\hline Non-professional & $457(27.9)$ \\
\hline Others & $943(57.5)$ \\
\hline \multicolumn{2}{|c|}{ Physical illness in the past 3 months } \\
\hline Yes & $331(20.1)$ \\
\hline No & 1318 (79.9) \\
\hline \multicolumn{2}{|l|}{ Sleep duration } \\
\hline$<7$ hours & $287(17.5)$ \\
\hline 7-8 hours & $1162(70.9)$ \\
\hline 9 hours or more & $190(11.6)$ \\
\hline \multicolumn{2}{|l|}{ Physical activity } \\
\hline Adequate & $140(10.1)$ \\
\hline Inadequate & 1245 (89.9) \\
\hline \multicolumn{2}{|l|}{ Depression } \\
\hline Moderate/severe & $248(14.8)$ \\
\hline Mild/normal & $1430(85.2)$ \\
\hline
\end{tabular}

*adjusted for the cluster sampling effect. health literacy level, 275 (16.4\%) respondents could be classified as adequate by the aforementioned definition (i.e. correct identification and intended to seek help).

The bivariate relationships between mental health literacy, demographics, familial variables, personal health conditions and behaviour, and depression were examined. The results were summarised in Table 3. As shown, MHL was significantly associated with depression without adjusting for other variables. There was an increased odds of about $60 \%$ of moderate to severe depression for those who had shown to have an inadequate level of MHL $(\mathrm{OR}=1.57,95 \%$ C.I $=1.03-2.39)$. None other variables in this sample were found to be significantly related to depression.

The results obtained from the multivariate linear regression analyses were presented in Table 4 . After adjusting for sex, mother's education level, and the cluster sampling effect, young people who had exhibited a moderate to severe level of depression in a week prior to the survey were more likely to have an inadequate level of MHL. The odds of depression was increased by about $60 \%$ for those who had an inadequate level of MHL (OR $=1.52$, 95\% C.I. $=1.01-2.31)$ in comparison to those who demonstrated an adequate level of MHL.

\section{Discussion}

This study aims to examine the relationship between mental health literacy and depression, in a population of Chinese adolescents. The results suggest that an inadequate mental health literacy level is significantly associated with moderate to severe level of depression as measured by the Depression sub-scale of the DASS. The point estimate prevalence of an adequate mental health literacy obtained from this study is low in comparison to those reported in the literature, particularly studies conducted in Australia by Jorm et al. [22,25] In one of their earlier studies in 2005 found that almost half of the respondents could not identify depression correctly [22]. However, in a later study in 2011 the rate of correct recognition of depression was found to be about 75\% [25]. Similar results on the recognition of depression symptoms were also found in another study carried out in Australia more recently. It was reported that $70 \%$ of higher education students were able to recognise depression in a vignette [29]. In comparison, only $23.4 \%$ of respondents in this study correctly identified the vignette as depression. The low rate of correct identification of depression could be related to the fact that the respondents were younger and might have less personal experience of depressive mood. It could also be related to the possibility that there is a lack of mental health education specifically designed to target young people in the geographic locality where the survey took place. In terms of the results obtained on the relationship between mental 
Table 2 Frequency and percentage of responses on some Mental Health Literacy variables $(\mathrm{N}=1678)$

MHL items
Recognition of disorder
Depression
Schizophrenia/paranoid schizophrenia
Psychosis/psychotic
Mental illness
Stress
Nervous breakdown
Psychological / mental / emotional problems
Has a problem
Cancer
Others
Nothing
Intended action to seek help
Yes
No/Don't know
Of those who seek help, seek help from**
Both parents
Mother
Father
Other people
Don't know

How confident in the ability to ask for help

Very confident

Fairly confident

Slightly confident

Not confident at all

Barrier of seeking help***

The costs of seeing the person

Person might feel negatively about you

What the person might say is wrong

Other people think of you seeing the person

Too far to travel

Too hard to get an appointment

Concern about side effect

Unlike the treatment offered

Thinking that nothing can help

Long wait for an appointment

Too embarrassed/shy

Other

If you have a similar problem, talk to parents

Yes

No/Don't know
Frequency (\%)*

$392(23.4 \%)$

$34(2.0 \%)$

7 (0.4\%)

$5(0.3 \%)$

555 (33.1\%)

34 (2.0\%)

459 27.4\%)

$60(3.6 \%)$

$11(0.7)$

42 (2.5\%)

77 (4.6\%)

1128 (67.7\%)

538 (32.3\%)

$653(57.1 \%)$

121 (10.6\%)

27 (2.4\%)

285 (24.9\%)

22 (1.9\%)

$20(1.7 \%)$

334 (29.8\%)

501 (44.7\%)

262 (23.4\%)

$23(2.1 \%)$

258 (23.1\%)

328 (29.4\%)

266 (23.8\%)

123 (11.0\%)

78 (7.0\%)

$105(9.4 \%)$

$163(14.6 \%)$

246 (22.0\%)

137 (12.3\%)

49 (4.4\%)

$330(29.6 \%)$

$71(6.4 \%)$

1047 (62.6\%)

626 (37.4\%)
Table 2 Frequency and percentage of responses on some Mental Health Literacy variables $(\mathbf{N}=\mathbf{1 6 7 8})$ (Continued)

Talk to both parents, mother or father 725 (67.8\%)

Both

$258(24.1 \%)$

Mother only

$37(3.5 \%)$

Father only

$49(435 \%)$

Don't know

Belief in First Aid

Would you help

Yes

$1097(65.7 \%)$

No

$574(34.4 \%)$

How confident be able to help

Very confident

$198(11.8 \%)$

Fairly confident

$677(40.4 \%)$

Slightly confident

$756(41.2 \%)$

Not confident at all

$43(2.6 \%)$

Helpful of the following:

Listening to the problem in an understanding way 1471 (879\%)

Talk firmly about getting act together

$1467(87.5 \%)$

Suggest to seek professional help

$1057(63.4 \%)$

Make an appointment to see a GP

$603(36.1 \%)$

Ask whether feeling suicidal

$379(22.7 \%)$

$67(4.0 \%)$

Suggest to have a few drinks to forget troubles

$1195(71.3 \%)$

Rally friends to cheer (him/her) up

$137(8.2 \%)$

$454(27.1 \%)$

$844(50.4 \%)$

Encourage to become more physically active

\section{Exposure to the problem}

Family or friends had a similar problem

Yes

$274(16.4 \%)$

No/Don't know

$1400(83.6 \%)$

The person received professional help**

$73(26.1 \%)$

Have you ever had a similar problem

Yes

$355(21.2 \%)$

No/Don't know

$1321(78.8 \%)$

You received professional help**

$54(15.3 \%)$

Treatment was helpful**

$75(30.9 \%)$

\section{Mental health literacy level}

Inadequate

$1403(83.6 \%)$

Adequate

$275(16.4 \%)$

*Percentage did not add up to $100 \%$ due to rounding; **Follow-up question only for those who answered yes in the previous question, ${ }^{* *}$ Multiple responses.

health literacy and depression among younger adolescents, comparison of results could be difficult since there has not been any other study found in the literature. Hence, this study could be considered as unique and the results obtained have not yet been reported before. 
Table 3 Unadjusted associations between mental health literacy level, other variables, and depression

\begin{tabular}{ll}
\hline Characteristics & \multicolumn{1}{c}{ Results* } \\
\hline Demographics & $X_{1}^{2}=0.68, p=0.501$ \\
Sex & $X_{1}^{2}=5.43, p=0.104$ \\
Age group & $X_{1}^{2}=2.02, p=0.088$ \\
Single child & $X_{1}^{2}=2.87, p=0.238$ \\
Family structure & $X_{2}^{2}=1.13, p=0.391$ \\
Father's education level & $X_{2}^{2}=1.40, p=0.583$ \\
Mother's education level & $X_{2}^{2}=0.74, p=0.681$ \\
Father's Occupation & $X_{2}^{2}=0.87, p=0.710$ \\
Mother's Occupation & $X_{1}^{2}=1.17, p=0.153$ \\
Physical illness in the past 3 months & \\
Health behaviours & $X_{1}^{2}=2.31, p=0.256$ \\
Physical activity & $X_{2}^{2}=3.98, p=0.215$ \\
Sleep duration & $X_{1}^{2}=4.68, p=0.039$ \\
Mental health literacy level &
\end{tabular}

The finding on the relationship between mental health literacy and the mental health status, among younger adolescents is important both in theoretical and practical senses. The results suggested that an inadequate mental health literacy level is associated with a higher level of depression symptoms. As defined in this study that the construct of mental health literacy incorporate two important components: first, the awareness of the problems as reflected from the correct identification of the mental health problem and, second, the attitude towards seeking appropriate help. An adequate level of mental health literacy is represented when both aspects are satisfied indicating that not only the individual acquire an understanding of the problem, but also having a positive attitude towards a help-seeking action. As aforementioned, lack of personal experience in depressive mood might be associated with the low rate of correct identification of the symptoms in the vignette. On the other hand, the results obtained suggest a low level of MHL is associated with depressive symptoms. A possible explanation for this seemingly contradictory phenomenon could be that young people who are in a depressive mood in this study, consciously or unconsciously, mislabel the symptoms as some other mental or physical health problems and incorrectly identify

Table 4 Adjusted Odd Ratios (95\% C.I.) of moderate to severe depression symptoms for mental health literacy levels

Variables retained in the model $\quad$ OR $(95 \%$ C.I.)*

Mental health literacy level

$1.00^{* *}$

Adequate

*Adjusted for sex, age, mother's education level, and cluster sampling effect; **Referent group. the vignette as well as showing a negative attitude towards help-seeking. This may due to a lack of knowledge and understanding of the symptoms they have experienced or the stigmatisation associated with any mental health problems, which is rather prominent in the region where the study took place. Should this be the case, the role of stigmatisation in the relationship between MHL and the mental health status would be an interesting area for further exploration. As being informed by different models of health behaviours, such as the Theory of Planned Behaviour, attitude plays a very important role in the implementation of the target health behaviour [37]. A positive attitude has a direct influence on the intention to perform the health behaviour and, in turn, induce the actual performance of the behaviour [37]. Applying this concept to the relationship between mental health literacy and mental health status that an inadequate level is associated with an increased risk of depression, one could understand that a correct identification of the problem in conjunction with a positive attitudes towards help-seeking would probably induce an actual behaviour in seeking appropriate help and thus resulting in a better mental health status.

The results obtained from this study have a direct implication on the early intervention of mental health problems, particularly depression among younger adolescents. As suggested by the results, an inadequate mental health literacy level is associated with a higher level of depression, thus enhancing the mental health literacy level of young people could be used as an early intervention measure of mental health problems. This echoes the conclusion drawn upon the results obtained from a systematic review of intervention programs for improving mental health literacy among young people by Jorm and his colleagues [38]. They concluded that a well-designed and evaluated program may lead to better mental health outcomes through the facilitation of early help-seeking [38]. From a population mental health perspective, more mental health literacy programs aiming for enhancing the understanding of mental health issues, fostering the correct attitudes towards help-seeking should be developed for young people, parents/carers, and teachers. Once their efficacy has been demonstrated, they should be implemented within the school system to maximise the benefits for young people. An example is the Youth Mental Health First Aids course for teachers developed and implemented in Australia with demonstrable results [39]. Similar programs could also be developed for young people with a specific linguistic and cultural focus for enhancing their mental health awareness in different countries such as China.

As in all studies, there are strengths and weaknesses in this study. This is a population-based study that includes a random sample of students from a large city utilising a 
two-stage cluster random sampling technique. An appropriate statistical analytical approach has been used to adjust for the effect of cluster sampling. The use of a standardised and validated assessment instrument for mental health literacy and psychological distress minimised some measurement biases for both the exposure and outcome variables. Some potential limitations have also been identified in this study. For example, a cross-sectional study could be considered as an appropriate design for exploring potential risk factors for a condition or disease. However, the evidence provided from such a study can only be considered as associative and is insufficient to draw any causal inference [40]. This study can be considered as an exploratory study to identify the potential association between mental health literacy and depression among adolescents. Furthermore, some important risk factors of depression among young people, such recent stressful life event and familial problems, were not included as potential confounder in the analysis. This may have caused a biased estimation of the strength of association between the exposure and outcome variables. Future studies could be conducted with a better design, such as a longitudinal cohort study, and to include the important potential confounding factors to elucidate whether the association is of a causal nature.

\section{Conclusion}

This study aims to investigate the relationship between mental health literacy and mental health status, particular depression of young people. The results indicated that mental health literacy was a potential factor that could have an impact on the mental health status of adolescents. Enhancing the mental health literacy level should be considered as an important preventive measure of mental health problems for young people.

\section{Competing interests}

The authors declare that they have no competing interests.

\section{Authors' contributions}

LTL is the principal investigator who formulated the research question, developed the study protocol, obtained institutional ethics approval, designed and piloted the survey questionnaire, conducted data analyses, and wrote the manuscript. The author read and approved the final manuscript.

\section{Acknowledgements}

The author would like to acknowledge the valuable assistance of Dr. Li Yang in supervising the field work during data collection.

Received: 29 April 2014 Accepted: 19 September 2014

Published: 25 September 2014

\section{References}

1. Institute of Medicine: Health literacy: a prescription to end confusion. Washington, DC: National Academies Press; 2004

2. $\mathrm{WHO}$, Commission on the Social Determinants of Health: Achieving health equity: From root causes to fair outcomes. Geneva: World Health Organisation 2007. Available on: http://whqlibdoc.who.int/publications/2007/interim _ statement_eng.pdf.

3. Jorm AF, Korten $A E$, Jacomb PA, Christensen $H$, Rodgers $B$, Pollitt $P$ : "Mental health literacy": a survey of the public's ability to recognise mental disorders and their belief about the effectiveness of treatment. Med J Aust 1997, 166:182-186.

4. Jorm AF: Mental health literacy: Public knowledge and beliefs about mental disorders. Br J Psychiatry 2000, 177:396-401.

5. McCann TV, Clark E: Australian Bachelor of Midwifery students' mental health literacy: an exploratory study. Nurs Health Sci 2010, 12:14-20.

6. Kermode M, Bowen K, Arole S, Joag K, Jorm AF: Community beliefs about causes and risks for mental disorders: a mental health literacy survey in a rural area of Maharashtra, India. Int J Soc Psychiatry 2010, 56:606-622.

7. Kermode M, Bowen K, Arole S, Joag K, Jorm AF: Community beliefs about treatments and outcomes of mental disorders: a mental health literacy survey in a rural area of Maharashtra, India. Public Health 2009, 123:476-483

8. Dahlberg KM, Waern M, Runeson B: Mental health literacy and attitudes in a Swedish community sample - investigating the role of personal experience of mental health care. BMC Public Health 2008, 8:8.

9. Yeo SG, Parker G, Yap HL, Mahendran R: Mental health literacy beliefs. A comparison of psychiatric trained nurses and enrolled nurses in Singapore. J Psychosoc Nurs Ment Health Serv 2003, 41:34-41.

10. Goldney RD, Fisher $\amalg$, Wilson DH, Cheok F: Mental health literacy of those with major depression and suicidal ideation: an impediment to help seeking. Suicide Life Threat Behav 2002, 32:394-403.

11. Chen H, Parker G, Kua J, Jorm A, Loh J: Mental health literacy in Singapore: a comparative survey of psychiatrists and primary health professionals. Ann Acad Med Singapore 2000, 29:467-473.

12. Jorm AF, Korten $A E$, Jacomb PA, Christensen $H$, Rodgers B, Pollitt P: Public beliefs about causes and risk factors for depression and schizophrenia. Soc Psychiatry Psychiatr Epidemiol 1997, 32:143-148.

13. Reavley NJ, Jorm AF: Recognition of mental disorders and beliefs about treatment and outcome: findings from an Australian national survey of mental health literacy and stigma. Aust N Z J Psychiatry 2011, 45:947-956

14. Reavley NJ, Jorm AF: Stigmatizing attitudes towards people with mental disorders: findings from an Australian National Survey of Mental Health Literacy and Stigma. Aust N Z J Psychiatry 2011, 45:1086-1093.

15. Jorm AF, Nakane Y, Christensen H, Yoshioka K, Griffiths KM, Wata Y: Public beliefs about treatment and outcome of mental disorders: a comparison of Australia and Japan. BMC Med 2005, 3:12

16. Griffiths KM, Christensen $\mathrm{H}$, Jorm AF: Mental health literacy as a function of remoteness of residence: an Australian national study. BMC Public Health 2009, 9:92.

17. Bartlett $H$, Travers $C$, Cartwright $C$, Smith N: Mental health literacy in rural Queensland: results of a community survey. Aust N Z J Psychiatry 2006, 40:783-789.

18. Wong DF, Xuesong H, Poon A, Lam AY: Depression literacy among Chinese in Shanghai, China: a comparison with Chinese-speaking Australians in Melbourne and Chinese in Hong Kong. Soc Psychiatry Psychiatr Epidemiol 2012, 47:1235-1242.

19. Wong FK, Lam YK, Poon A: Depression literacy among Australians of Chinesespeaking background in Melbourne, Australia. BMC Psychiatry 2010, 10:7.

20. Wong DF, Xuesong H: Schizophrenia literacy among Chinese in Shanghai, China: a comparison with Chinese-speaking Australians in Melbourne and Chinese in Hong Kong. Aust N Z J Psychiatry 2011, 45:524-31.

21. Tieu $Y$, Konnert $C$, Wang J: Depression literacy among older Chinese immigrants in Canada: a comparison with a population-based survey. Int Psychogeriatr 2010, 22:1318-1326.

22. Wright A, Harris MG, Wiggers JH, Jorm AF, Cotton SM, Harrigan SM, Hurworth RE, McGorry PD: Recognition of depression and psychosis by young Australians and their beliefs about treatment. Med J Aust 2005, 183:18-23.

23. Burns JR, Rapee RM: Adolescent mental health literacy: young people's knowledge of depression and help seeking. J Adolesc 2006, 29:225-239.

24. Leighton S: Using a vignette-based questionnaire to explore adolescents' understanding of mental health issues. Clin Child Psychol Psychiatry 2010, 15:231-250

25. Reavley NJ, Jorm AF: Young people's recognition of mental disorders and beliefs about treatment and outcome: findings from an Australian national survey. Aust N Z J Psychiatry 2011, 45:890-898.

26. Vanheusden $\mathrm{K}$, Mulder $\mathrm{CL}$, van der Ende J, van Lenthe FJ, Mackenbach JP, Verhulst FC: Young adults face major barriers to seeking help from mental health services. Patient Educ Couns 2008, 73:97-104.

27. Khan TM, Sulaiman SA, Hassali MA: Mental health literacy towards depression among non-medical students at a Malaysian university. Ment Health Fam Med 2010, 7:27-35. 
28. Reavley NJ, McCann TV, Jorm AF: Actions taken to deal with mental health problems in Australian higher education students. Early Interv Psychiatry 2012, 6:159-165.

29. Reavley NJ, McCann TV, Jorm AF: Mental health literacy in higher education students. Early Interv Psychiatry 2012, 6:45-52.

30. McCann TV, Lu S, Berryman C: Mental health literacy of Australian Bachelor of Nursing students: a longitudinal study. J Psychiatr Ment Health Nurs 2009, 16:61-67.

31. Lawlor E, Breslin JG, Renwick L, Foley S, Mulkerrin U, Kinsella A, Turner N, O'Callaghan E: Mental health literacy among Internet users. Early Interv Psychiatry 2008, 2:247-55.

32. Eckert KA, Kutek SM, Dunn KI, Air TM, Goldney RD: Changes in depression-related mental health literacy in young men from rural and urban South Australia. Aust J Rural Health 2010, 18:153-158.

33. Addington D, Berzins S, Yeo M: Psychosis literacy in a Canadian health region: results from a general population sample. Can J Psychiatry 2012, 57:381-388.

34. Antony MM, Bieling PJ, Cox BJ, Enns MW, Swinson RP: Psychometric properties of the 42-item and 21-item versions of the Depression Anxiety Stress Scales in clinical groups and a community sample. Psychol Assess 1988, 10:176-181.

35. Szabó M, Lovibond PF: Anxiety, depression and tension/stress in children. Psychopathol Behav Assess 2006, 28:195-205.

36. World Health Organisation: Global recommendations on physical activity for health. Geneva: WHO; 2010

37. Ajzen I, Driver BL: Prediction of leisure participation from behavioural, normative, and control beliefs: An application of the Theory of Planned Behaviour. Leisure Sci 1991, 13:185-204.

38. Kelly CM, Jorm AF, Wright A: Improving mental health literacy as a strategy to facilitate early intervention for mental disorders. Med J Aust 2007, 187:\$26-30.

39. Jorm AF, Kitchener BA, Sawyer MG, Scales H, Cvetkovski S: Mental health first aid training for high school teachers: A cluster randomised trial. BMC Psychiatry 2010, 10:1-12.

40. Rothman KJ, Greenland S: Morden Epidemiology $2^{\text {nd }}$ Ed. Philadelphia, PA: Lippincott Williams \& Wilkins; 1998.

\section{Submit your next manuscript to BioMed Central and take full advantage of:}

- Convenient online submission

- Thorough peer review

- No space constraints or color figure charges

- Immediate publication on acceptance

- Inclusion in PubMed, CAS, Scopus and Google Scholar

- Research which is freely available for redistribution 